УДК 621.313 .33

\title{
МАТЕМАТИЧЕСКАЯ МОДЕЛЬ АСИНХРОННОГО ДВИГАТЕЛЯ В МУЛЬТИФАЗНОЙ СИСТЕМЕ КООРДИНАТ ПРИ НЕСИММЕТРИИ РОТОРНЫХ ЦЕПЕЙ
}

\author{
Глазырин Александр Савельевич1,2, \\ asglazyrin@tpu.ru
}

Полищук Владимир Иосифович ${ }^{3}$, polischuk_vi@mail.ru

Тимошкин Вадим Владимирович1, tim_vv@mail.ru

\author{
Баннов Дмитрий Михайлович4, \\ bannov.dm@samgtu.ru
}

Исаев Юсуп Ниязбекович'1, isaev_yusup@mail.ru

\section{Антяскин Дмитрий Ильич 5 ,} antyaskin.dmitriy@gmail.com

\author{
Кладиев Сергей Николаевич1, \\ kladiev@tpu.ru
}

Филипас Александр Александрович 1 , filipas@tpu.ru
Ланграф Сергей Владимировичє, sergeylgrf@gmail.com

\author{
Котин Денис Алексеевич 7 , \\ d.kotin@corp.nstu.ru
}

\section{Ковалев Владимир Захарович2,} vz_kovalev@mail.ru

1 Национальный исследовательский Томский политехнический университет, Россия, 634050, г. Томск, пр. Ленина, 30.

2 Югорский государственный университет, Россия, 628012, г. Ханты-Мансийск, ул. Чехова, 16.

3 Алтайский государственный технический университет им. И.И. Ползунова, Россия, 656038, Алтайский край, г. Барнаул, пр. Ленина, 46.

4 Самарский государственный технический университет, Россия, 443100, г. Самара, ул. Молодогвардейская, 224.

5000 «Комплексные автоматизированные системы», Россия, 634021, г. Томск, пр. Фрунзе, 240а.

6000 «НПФ Мехатроника-Про», Россия, 634021, г. Томск, пр. Фрунзе, $119 \mathrm{E}$

7 Новосибирский государственный технический университет, Россия, 630073, г. Новосибирск, пр. К. Маркса, 20.

\begin{abstract}
Актуальность исследования обусловлена острой необходимостью в теоретическом обосновании и практической разработке селективных методов диагностирования сложных внутренних повреждений мощных высоковольтных асинхронных двигателей с короткозамкнутьмм ротором, являющихся одним из главных элементов ответственных механизмов всех технологических процессов в топливноэнергетическом комплексе. Как правило, спецификой работы высоковольтных асинхронных двигателей с короткозамкнутьмм ротором в топливно-энергетическом комплексе как на стадии добьчи и транспортировки георесурсов, так и на стадии их переработки являются тяжелые условия пуска, особенно это выражено для ответственных механизмов тепловых электростанций (питательные насосы, мельницы, дробилки, дымососы, дутьевые вентиляторы и т. д.). Отказы асинхронных двигателей с короткозамкнутым ротором на тепловых электростанциях приводят либо к отключению энергоблока, либо как минимум к снижению уровня выработки электроэнергии. При этом, несмотря на тяжелейшие и, как правило, необратимые последствия от такого повреждения - десбекты в короткозамкнутой обмотке ротора асинхронных двигателей - защит от данного повреждения не существует, и выявляется оно только в период капитального ремонта. Обрыв стержня ротора, вызывающий несимметрию роторных иепей, в начальной стадии носит скрытый характер и является толчком для развития более опасных аварийных режимов. Отсутствие апробированных технических средств диагностики данного вида повреждения, прежде всего, связано с недостаточной фоормализаиией математического описания процессов в асинхронных двигателях с короткозамкнутым ротором при возникновении дефректов в обмотке ротора.

Объект: высоковольтные асинхронные электродвигатели переменного тока с короткозамкнутым ротором.

Цель: разработать математическую модель асинхронной машины с несимметрией роторных цепей, адекватно отображающую фризические процессы в машине при возникновении повреждений в обмотке ротора.

Методы и средства. Для достижения поставленной цели применялись теоретические методы исследований. К ним относятся: теория электрических машин, численные методы. Имитационное моделирование производилось в среде MatLab, a математическая обработка данных - в пакете MathCad.

Результаты. Разработана п-фразная имитационная модель асинхронного двигателя, позволяющая исследовать обрыв стержня в беличьей клетке. Предложены аналитические выражения, которые описывают процессы в двигателе при неподвижном роторе.
\end{abstract}

Ключевые слова:

Асинхронный двигатель, математическая модель, «беличья клетка», обрыв стержня ротора, диагностика. 


\section{Введение}

Во всем мире в электроприводах всех ответственных механизмов собственных нужд (CH) тепловых электрических станций (ТЭС) используются асинхронные двигатели с короткозамкнутым ротором (АДКР). В России ТЭС, работающие на угле и газе, вырабатывают более половины всей электроэнергии, и согласно стратегии развития электроэнергетики, их количество будет неуклонно расти. Если учесть, что совершенствование технологических процессов неизбежно увеличивает коэффициент использования АДКР ответственных механизмов СН ТЭС, то со временем неизбежно будет возрастать число отказов ответственных механизмов СН ТЭС по вине АДКР.

Высоковольтные АДКР на ТЭС, как правило, работают в тяжелых условиях пуска, и у них наблюдаются частые повреждения обмоток. Из числа всех возникающих у АДКР дефектов на обмотку ротора приходится от 11 до 17 \% в зависимости от типа машин $[1,2]$. К самым распространенным повреждениям ротора относятся обрывы стержней «беличьей клетки», что составляет порядка $80 \%$ от числа дефектов в роторной цепи [1]. Обрыв стержня обмотки ротора выявляют в основном при капитальном ремонте АДКР. В то же время из-за большой периодичности капитальных ремонтов такие дефекты своевременно не определяются, и АДКР может длительно работать с трещинами в стержнях, при этом потребляя дополнительную энергию и выделяя больше тепла в пусковых и в установившихся режимах [2]. Во время эксплуатации таких АДКР возрастает потребляемая ими мощность и уровень вибрации, и вследствие воздействия на стержень центробежных сил он отгибается и задевает магнитопровод статора, что полностью выводит из строя двигатель и его крайне затруднительно восстановить.

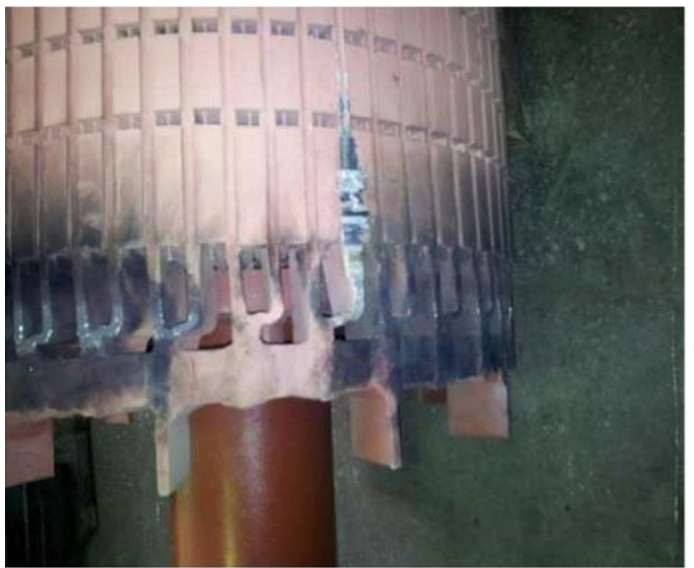

$\mathrm{a}(\mathrm{a})$

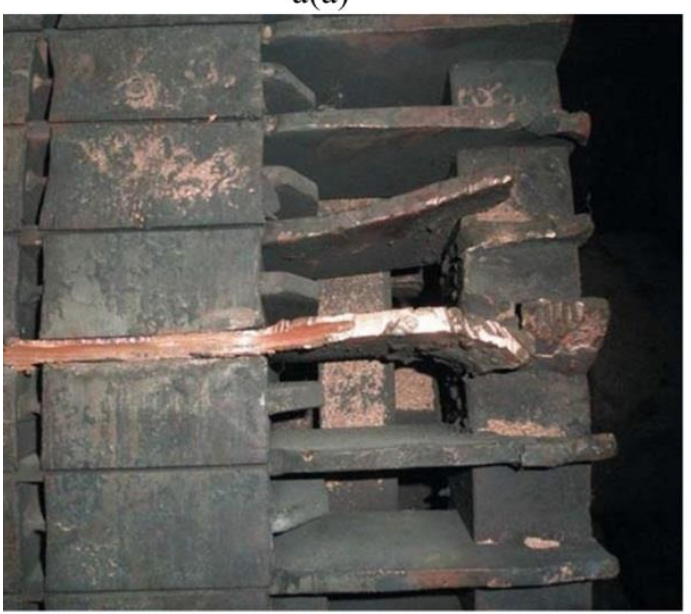

$\mathrm{B}(\mathrm{c})$

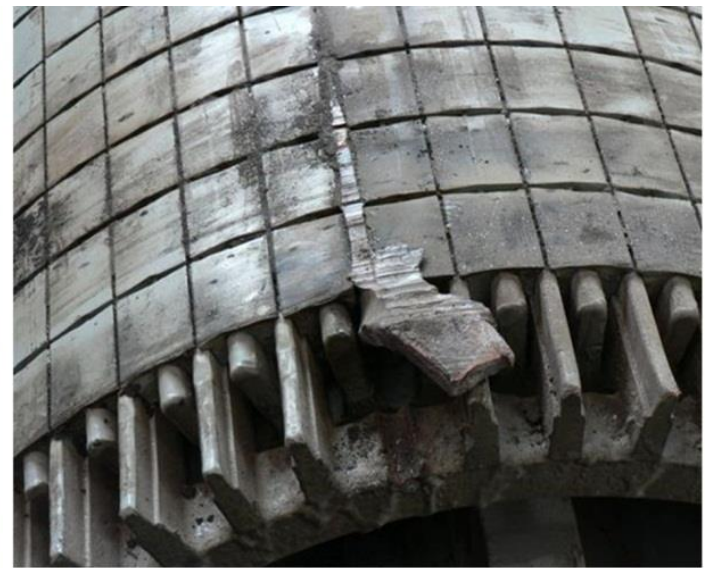

б(b)

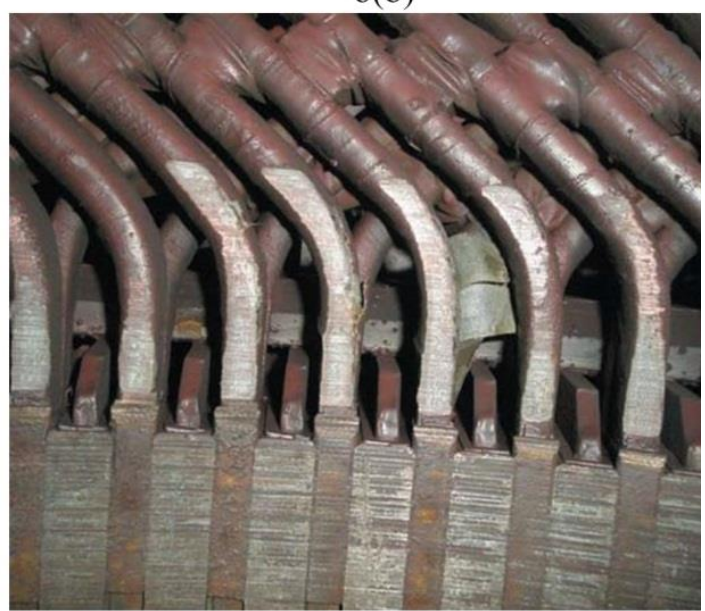

$\Gamma(\mathrm{d})$

Рис. 1. Последствия обрыва стержня ротора высоковольтного асинхронного двигателя с короткозамкнутым ротором: а) выход стержня из паза ротора асинхронного двигателя типа АДЧР-1600 с последующим коротким замыканием между фазами и на землю статорной обмотки; б) выход стержня из паза ротора асинхронного двигателя типа ДАЗО; в) выход стержня из паза ротора асинхронного двигателя типа АНЗ; г) повреждение активной части статора высоковольтного асинхронного двигателя с короткозамкутым ротором типа АH3

Fig. 1. Consequences of high voltage squirrel-cage rotor bar breakage: a) core exit from rotor slot of squirrel-cage induction motor ADCHR-1600 type with subsequent short circuit between phases and to ground of stator winding; $b$ ) core exit from rotor slot of squirrel-cage induction motor DAZO type; c) core exit from rotor slot of squirrel-cage induction motor ANZ type; d) damage of active part of stator of high voltage squirrel-cage induction motor ANZ type 
На рис. 1 показаны последствия выхода из паза оторванного стержня, повлекшие за собой необходимость капитального ремонта лобовых частей статорной обмотки и магнитопровода. Как видно в первом случае, оторванный стержень нарушает изоляцию, замыкая токопроводящие части между собой и на корпус, чему свидетельствуют следы выделения большого количества тепловой энергии на внешней стороне ротора. На сегодняшний день защит, обладающих достаточной чувствительностью, способной определять дефект обмотки ротора АДКР, не существует. А методы диагностики такого рода неисправностей АДКР, основанные на термографическом, вибрационном, визуальном анализе [3-10], не дают достоверных и селективных диагностических признаков. При этом методы компенсации влияния электрической несимметрии на механический момент асинхронного двигателя комплексно не приносят желаемого эффекта в связи с неполной эффективностью и сложностью реализации [9]. Поэтому задача по разработке адекватной математической модели АДКР, позволяющей оценить разницу между бездефектным состоянием ротора и поврежденным, всегда являлась актуальной.

\section{Разработка $n$-фразной математической модели асинхронного двигателя}

Для исследования обрыва стержней в роторе необходима математическая модель АДКР, которая должна удовлетворять ряду требований: возможность задавать количество стержней в роторе, описывать адекватно процессы, возникающие в электрической машине, при нормальном и аварийном состоянии.

Рассмотрим описание электрической машины в $n$ фазной системе координат, где $n$ сответствует общему количеству стержней в роторе. Процессы в роторе и статоре будем описывать в системе координат с одинаковой размерностью.

Асинхронный электродвигатель можно представить в виде эквивалентной многоконтурной схемы замещения, где каждый контур имеет свое активное сопротивление, индуктивности рассеяния и взаимные индуктивности. Изменяя соответствующее сопротивление $R_{R}{ }^{(1)}$ в контуре ротора, можно моделировать повреждение стержня (рис. 2).

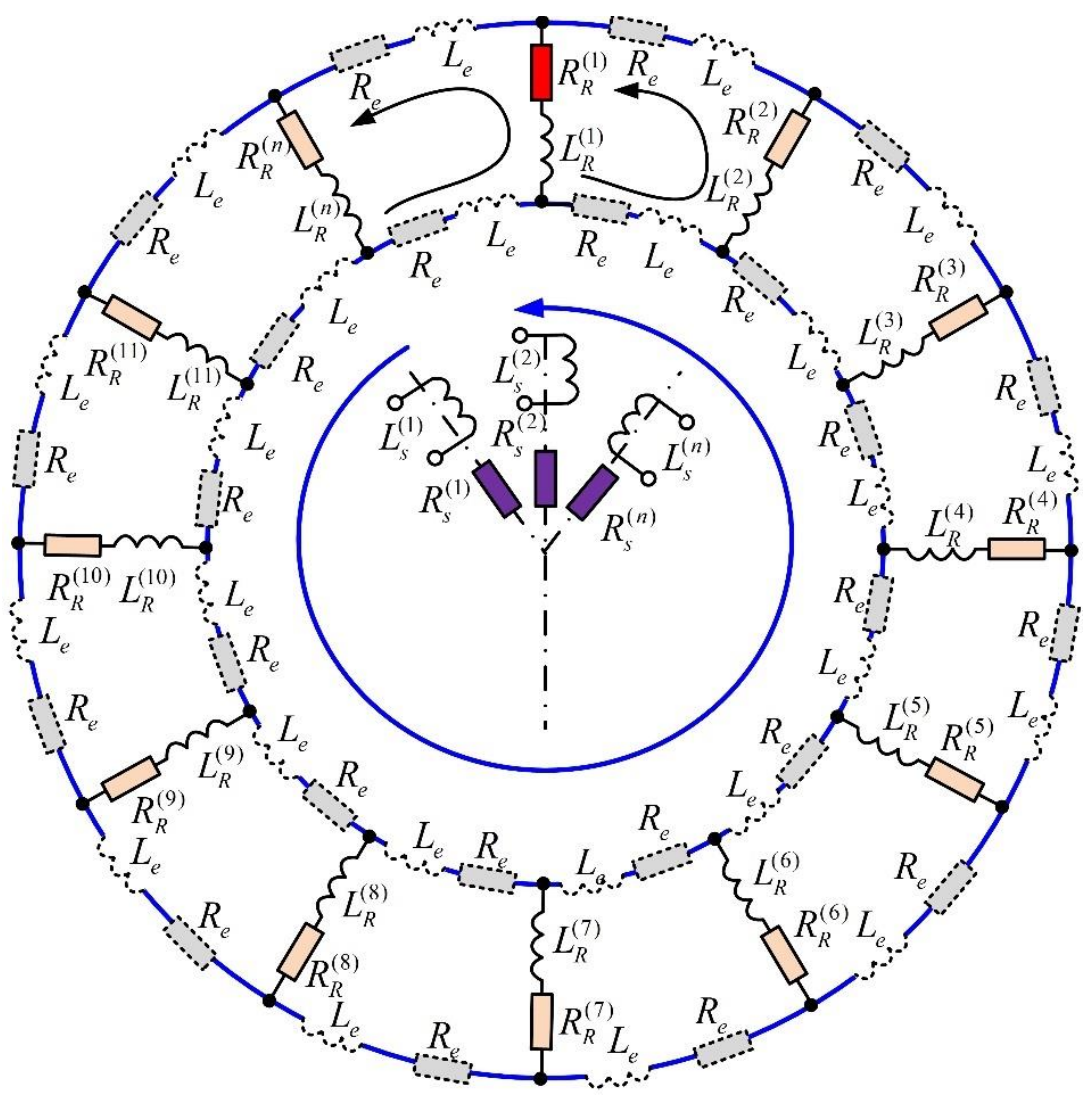

Puc. 2. Эквивалентная схема ротора и статора

Fig. 2. Equivalent rotor and stator circuit

При рассмотрении $n$-фазной машины было сделано допущение, которое позволяет упростить математическое описание и не учитывать комплексное сопротивление $\left(R_{e}+j \omega L_{e}\right)$ секторов короткозамкнутого кольца между соседними стержнями ротора (рис. 2).
Для построения $n$-фазной математической модели будем использовать общеизвестные принципы и выражения, которые применяются при моделировании асинхронных машин [11-15].

Система дифференциальных уравнений для $n$ фазной машины будет иметь вид: 


$$
\left\{\begin{array}{l}
\frac{d \psi_{S}^{(1)}}{d t}=U^{(1)}-R_{S} \cdot i_{S}^{(1)} \\
\cdot \cdot \\
\frac{d \psi_{S}^{(n)}}{d t}=U^{(n)}-R_{S} \cdot i_{S}^{(n)} \\
\frac{d \psi_{R}^{(1)}}{d t}=0-R_{R} \cdot i_{R}^{(1)} ; \\
\cdot \cdot \\
\frac{d \psi_{R}^{(n)}}{d t}=0-R_{R} \cdot i_{R}^{(n)},
\end{array}\right.
$$

где $R_{S}$ - активное сопротивление статора; $R_{R}$ - активное сопротивление ротора; $i_{S}$ - ток статора; $i_{R}-$ ток ротора; $\psi_{S}$ - потокосцепление статора; $\psi_{R}$ - потокосцепление ротора; $U$ - входное напряжение для статорной обмотки.

Из представленной системы дифференциальных уравнений (1) видно, что $n$ определяет количество стержней. С увеличением стержней в роторе порядок системы повышается, что усложняет расчеты.

Разрабатываемая математическая модель предназначена для исследования асинхронного двигателя с короткозамкнутой обмоткой при обрыве стрежней. Вектор входных напряжений формируется из составляющих статора и ротора, но так как двигатель имеет короткозамкнутую обмотку, то входные воздействия для роторной цепи будут всегда равны нулю.

Вектор входных напряжений будет формироваться следующим образом:

$$
U_{\mathrm{BX}}=\left[U^{(1)} U^{(2)} . . U^{(n)} 0 \ldots 0^{(n)}\right]^{T} .
$$

Напряжения в многомерной системе координат описываются по следующему выражению:

$$
U^{(n)}(t)=U_{m} \cdot \cos \left(\theta+\varphi_{\text {сд }}\right),
$$

где $U_{m}$ - амплитудное значение, $\theta=2 \pi f t=\omega t ; \varphi_{\text {сд }}-$ фазовый угол сдвига.

Зададимся напряжениями в трехфазной системе:

$$
\begin{gathered}
U_{A}(t)=\sqrt{2} U_{A} \sin (\omega \cdot t), \\
U_{B}(t)=\sqrt{2} U_{B} \sin \left(\omega \cdot t-\frac{2 \pi}{3}\right), \\
U_{C}(t)=\sqrt{2} U_{C} \sin \left(\omega \cdot t+\frac{2 \pi}{3}\right),
\end{gathered}
$$

где $U_{A}, U_{B}, U_{C}$ - действующие значения напряжений.

Амплитудное значение напряжения для каждой фазы статора не зависит от системы координат:

$$
U_{m}=\sqrt{U_{A}^{2}(t)+\frac{\left(U_{B}(t)-U_{C}(t)\right)^{2}}{3}}=\sqrt{2} \cdot U,
$$

где $U$ - действующее значение напряжения для каждой фазы статора.

Угол $\theta$ определим из уравнений напряжений в трехфазной системе координат:

$$
\theta=\arctan \left(\frac{U_{A}(t) \sqrt{3}}{U_{B}(t)-U_{C}(t)}\right) .
$$

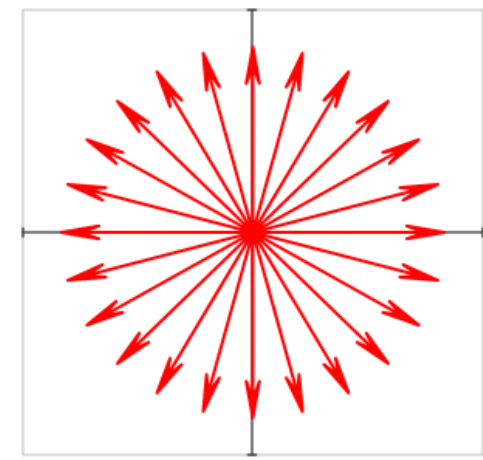

Рис. 3. Векторная диаграмма

Fig. 3. Vector diagram

Угол сдвига между составляющими напряжений в многомерной системе будет зависеть от числа стержней $n$, как показано на рис. 3:

$$
\varphi_{\text {сд }}=\frac{2 \cdot \pi}{n} .
$$

Далее, согласно закону Ампера, находим токи в статоре и роторе:

$$
\left[\begin{array}{l}
\bar{\psi}_{S} \\
\bar{\psi}_{R}
\end{array}\right]=L_{0}\left[\begin{array}{l}
\bar{I}_{S} \\
\bar{I}_{R}
\end{array}\right] \Rightarrow\left[\begin{array}{l}
\bar{I}_{S} \\
\bar{I}_{R}
\end{array}\right]=L_{0}^{-1} \cdot\left[\begin{array}{l}
\bar{\psi}_{S} \\
\bar{\psi}_{R}
\end{array}\right],
$$

где $L_{0}=\left[\begin{array}{ll}L_{S 0} & L_{S R} \\ L_{R S} & L_{R 0}\end{array}\right]$ - матрица индуктивностей; $L_{S 0}-$ собственная матрица индуктивностей для статора; $L_{R 0}$ - собственная матрица индуктивностей для ротоpa; $L_{S R}, L_{R S}-$ матрицы взаимных индуктивностей; $\bar{\psi}_{S}=\left[\begin{array}{c}\psi_{S}^{(1)} \\ : \\ \psi_{S}^{(n)}\end{array}\right] \quad-\quad$ вектор потокосцепления статора; $\bar{\psi}_{R}=\left[\begin{array}{c}\psi_{R}^{(1)} \\ : \\ \psi_{R}^{(n)}\end{array}\right]-$ вектор потокосцепления ротора; $\bar{I}_{S}=\left[\begin{array}{c}I_{S}^{(1)} \\ : \\ I_{S}^{(n)}\end{array}\right]-$ вектор тока статора; $\bar{I}_{R}=\left[\begin{array}{c}I_{R}^{(1)} \\ : \\ I_{R}^{(n)}\end{array}\right]-$ вектор тока ротора.

Для определения собственных матриц индуктивностей статора и ротора используется вспомогательная матрица коэффициентов $\operatorname{Corr}(\varphi)$, которая имеет зависимость от $\varphi$ :

$$
\begin{gathered}
\operatorname{Corr}(\varphi)= \\
=\left[\begin{array}{ccccc}
0 & \cos (\varphi) & \cos (2 \varphi) & . . & \cos (-a \varphi) \\
\cos (-\varphi) & 0 & \cos (\varphi) & . . & \cos (-b \varphi) \\
\cos (-2 \varphi) & \cos (-\varphi) & 0 & . . & \cos (-c \varphi) \\
: & : & : & . . & : \\
\cos (-a \varphi) & \cos (-b \varphi) & \cos (-c \varphi) & . . & 0
\end{array}\right], \\
a=(n-1) ; b=(n-2) ; c=(n-3) .
\end{gathered}
$$

Матрица собственных индуктивностей статора: $L_{s 0}=L_{m} \cdot \operatorname{Corr}(\varphi)+\operatorname{diag}\left[L_{S}^{(1)}, L_{S}^{(2)} \ldots L_{S}^{(n)}\right]$, 
где $L_{m}$ - максимальная приведенная взаимная индуктивность между фазами статора и ротора.

Матрица собственных индуктивностей ротора:

$$
L_{R 0}=L_{m} \cdot \operatorname{Corr}(\varphi)+\operatorname{diag}\left[L_{R}^{(1)}, L_{R}^{(2)} \ldots L_{R}^{(n)}\right] .
$$

Вычисление матриц $L_{S 0}$ и $L_{R 0}$ не требует значительных вычислительных мощностей, так как нет зависимости от угла поворота ротора.

Элементы $L_{S R}$ и $L_{R S}$ задаются также с помощью вспомогательной матрицы, которая имеет зависимость от $\varphi$ и $\gamma$ :

$$
\begin{gathered}
\operatorname{Cos}(\gamma, \varphi)= \\
=\left[\begin{array}{cccc}
\cos (b) & \cos (b-\varphi) & . . & \cos (b-a \cdot \varphi) \\
\cos (b-\varphi) & \cos (b-\varphi-\varphi) & . . & \cos (b-a) \cdot \varphi-\varphi) \\
: & : & . . & : \\
\cos (b-a \varphi) & \cos (b-\varphi-a \varphi) & . . & \cos (b-a \varphi-a \varphi)
\end{array}\right], \\
a=(n-1) ; b=\gamma_{e}
\end{gathered}
$$

где $\frac{d \gamma_{e}}{d t}=z_{p} \int \omega_{r} d t-$ угол поворота ротора.

Окончательные выражения для определения $L_{S R}$ и $L_{R S}$ имеют вид:

$$
\begin{gathered}
L_{R S}=L_{m} \cdot \operatorname{Cosr}(\gamma, \varphi), \\
L_{S R}=L_{m} \cdot \operatorname{Cosr}(\gamma, \varphi)^{T} .
\end{gathered}
$$

После всех преобразований получаем систему дифференциальных уравнений:

$$
\left[\begin{array}{c}
\frac{d \psi_{S}^{(1)}}{d t} \\
: \\
\frac{d \psi_{S}^{(n)}}{d t} \\
\frac{d \psi_{R}^{(1)}}{d t} \\
: \\
\frac{d \psi_{R}^{(n)}}{d t}
\end{array}\right]=\left[\begin{array}{c}
U^{(1)} \\
: \\
U^{(n)} \\
0 \\
: \\
0^{(n)}
\end{array}\right]-R \cdot L_{0}^{-1} \cdot\left[\begin{array}{c}
\psi_{S}^{(1)} \\
: \\
\psi_{S}^{(n)} \\
\psi_{R}^{(1)} \\
: \\
\psi_{R}^{(n)}
\end{array}\right],
$$

где $R=\left[\begin{array}{cc}R_{S 0} & 0 \\ 0 & R_{R 0}\end{array}\right]$ - главная матрица сопротивлений; $R_{s 0}=\operatorname{diag}\left[R_{S}^{(1)}, R_{S}^{(2)} . . R_{S}^{(n)}\right]-$ матрица сопротивлений статора; $R_{R 0}=\operatorname{diag}\left[R_{R}^{(1)}, R_{R}^{(2)} . . R_{R}^{(n)}\right]$ - матрица сопротивлений ротора.

Электромагнитный момент асинхронной машины определяется из токов статора и ротора:

$$
M_{\text {дв }}=-z_{p} \cdot \bar{I}_{S}^{T} \cdot L_{n} \cdot \bar{I}_{R} \cdot \frac{3}{n},
$$

где $z_{p}$ - число пар полюсов;

$$
=\left[\begin{array}{cccc}
\sin (b) & \sin (b-\varphi)= & \\
\sin (b-\varphi) & \sin (b-\varphi-\varphi) & . . & \sin (b-a \varphi-\varphi) \\
. . & . . & . . & . . \\
\sin (b-a \varphi) & \sin (b-\varphi-a \varphi) & . . & \sin (b-a) \varphi-a \varphi)
\end{array}\right]
$$

- вспомогательная матрица;

$$
L_{n}=\frac{d\left[L_{R S}\right]}{d \gamma_{e}}=-s\left(\varphi, \gamma_{e}\right) \cdot L_{m} .
$$

Для обеспечения необходимой мощности на валу двигателя в $n$-фазной системе координат вводится коэффициент $3 / n$, который позволяет независимо от размерности системы дифференциальных уравнений обеспечивать паспортный момент двигателя.

Математическое описание механической части асинхронной машины строится на базе одномассовой системы:

$$
M_{\text {дв }}-M_{C}=J \frac{d \omega}{d t},
$$

где $J$ - момент инерции двигателя; $M_{C}$ - момент нагрузки.

Имитация обрыва стержней в данном математическом описании реализуется с помощью введения добавочного сопротивления в матрицу $R_{R 0}$ для соответствующего стержня.

Для диагностики обрыва стержней в роторе используется модуль результирующего вектора тока статора (огибающая токов статора):

$$
I_{m 1}(t)=\sqrt{I_{S}^{(1)}(t)+I_{S}^{(2)}(t)+I_{S}^{(3)}(t)+\ldots I_{S}^{(n)}(t)},
$$

где $I_{S}^{(1)}(t)$ - фазный ток статора.

Согласование огибающей токов статора в $n$ фазной системе координат с трехфазной реализуется с помощью масштабирующего коэффициента $k$, который зависит от размерности системы:

$$
k=\sqrt{\frac{3}{n}} .
$$

Значение огибающей токов статора в трехфазной системе можно найти по выражению:

$$
I_{m}(t)=I_{m 1}(t) \cdot k,
$$

где $I_{m 1}(t)$ - огибающая токов статора в многофазной системе.

\section{Математическое описание трехфазной машины \\ при неподвижном роторе}

Асинхронные машины можно исследовать и при неподвижном роторе. При нулевой угловой скорости ротора математическое описание значительно упрощается и есть возможность получить аналитические выражения. Так как режим покоя для $n$-фазной модели является частным случаем, то полученные результаты можно использовать для подтверждения ее работоспособности.

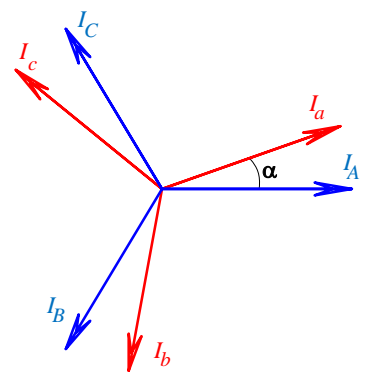

Pис. 4. Векторная диаграмма токов статора и ротора

Fig. 4. Vector diagram of stator and rotor currents 
Рассмотрим синтез системы уравнений, позволяющий получить в аналитическом виде описание токов для трехфазной машины при неподвижном роторе и с углами между обмотками статора и ротора (рис. 4):

$\alpha=0, \cos (\alpha), \cos (\alpha+2 \pi / 3)=-1 / 2$,

$$
\cos (\alpha-2 \pi / 3)=-1 / 2 \text {. }
$$

Взаимные индуктивности при этом составляют:

$$
\begin{gathered}
M_{A a}=M_{a A}=L_{m} \cos (\alpha)=L_{m}, \\
M_{A b}=M_{b A}=L_{m} \cos (\alpha+2 \pi / 3)=-1 / 2 L_{m}, \\
M_{A c}=M_{b A}=L_{m} \cos (\alpha-2 \pi / 3)=-1 / 2 L_{m} .
\end{gathered}
$$

Учитываем влияние взаимной индуктивности обмоток статора и обмоток ротора и получаем систему дифференциальных уравнений для обмоток ротора:

$$
\left\{\begin{array}{l}
L_{R} \frac{d i_{a}}{d t}+i_{a} R_{R}-\frac{1}{2} L_{m}\left(\frac{d i_{B}}{d t}+\frac{d i_{C}}{d t}\right)+ \\
+L_{m}\left(\frac{d i_{A}}{d t}-\frac{1}{2} \frac{d i_{B}}{d t}-\frac{1}{2} \frac{d i_{C}}{d t}\right)=0 \\
L_{R} \frac{d i_{b}}{d t}+i_{b} R_{R}-\frac{1}{2} L_{m}\left(\frac{d i_{A}}{d t}+\frac{d i_{C}}{d t}\right)+ \\
+L_{m}\left(\frac{d i_{B}}{d t}-\frac{1}{2} \frac{d i_{A}}{d t}-\frac{1}{2} \frac{d i_{C}}{d t}\right)=0 \\
L_{R} \frac{d i_{c}}{d t}+i_{c} R_{R}-\frac{1}{2} L_{m}\left(\frac{d i_{B}}{d t}+\frac{d i_{A}}{d t}\right)+ \\
+L_{m}\left(\frac{d i_{C}}{d t}-\frac{1}{2} \frac{d i_{B}}{d t}-\frac{1}{2} \frac{d i_{A}}{d t}\right)=0
\end{array}\right.
$$

и уравнения для обмоток статора:

$$
\left\{\begin{array}{l}
L_{S} \frac{d i_{A}}{d t}+i_{A} R_{S}-\frac{1}{2} L_{m}\left(\frac{d i_{B}}{d t}+\frac{d i_{C}}{d t}\right)+ \\
+L_{m}\left(\frac{d i_{a}}{d t}-\frac{1}{2} \frac{d i_{b}}{d t}-\frac{1}{2} \frac{d i_{c}}{d t}\right)=U_{A}(t) ; \\
L_{S} \frac{d i_{B}}{d t}+i_{B} R_{S}-\frac{1}{2} L_{m}\left(\frac{d i_{A}}{d t}+\frac{d i_{C}}{d t}\right)+ \\
+L_{m}\left(\frac{d i_{b}}{d t}-\frac{1}{2} \frac{d i_{a}}{d t}-\frac{1}{2} \frac{d i_{c}}{d t}\right)=U_{B}(t) ; \\
L_{S} \frac{d i_{C}}{d t}+i_{C} R_{S}-\frac{1}{2} L_{m}\left(\frac{d i_{B}}{d t}+\frac{d i_{C}}{d t}\right)+ \\
+L_{m}\left(\frac{d i_{c}}{d t}-\frac{1}{2} \frac{d i_{b}}{d t}-\frac{1}{2} \frac{d i_{c}}{d t}\right)=U_{C}(t) .
\end{array}\right.
$$

Здесь индексы $R$ и $S$ означают принадлежность сопротивлений и индуктивностей к ротору и статору соответственно.

Разрешим системы уравнений (11) и (12) относительно производных и запишем в нормальной форме Коши:

$$
\left(\begin{array}{l}
d i_{a} / d t \\
d i_{b} / d t \\
d i_{c} / d t \\
d i_{A} / d t \\
d i_{B} / d t \\
d i_{C} / d t
\end{array}\right)=\mathbf{A}\left(\begin{array}{c}
i_{a} \\
i_{b} \\
i_{c} \\
i_{A} \\
i_{B} \\
i_{C}
\end{array}\right)+\mathbf{B}
$$

или в развернутом виде

$$
\left(\begin{array}{l}
d i_{a} / d t \\
d i_{b} / d t \\
d i_{c} / d t \\
d i_{A} / d t \\
d i_{B} / d t \\
d i_{C} / d t
\end{array}\right)=\left(\begin{array}{ll}
\mathbf{A}_{11} & \mathbf{A}_{12} \\
\mathbf{A}_{21} & \mathbf{A}_{22}
\end{array}\right)\left(\begin{array}{l}
i_{a} \\
i_{b} \\
i_{c} \\
i_{A} \\
i_{B} \\
i_{C}
\end{array}\right)+\mathbf{B},
$$

где

$$
\begin{gathered}
\mathbf{B}=\frac{1}{2 L_{S} L_{R}+L_{m}\left(L_{R}+L_{S}-4 L_{m}\right)} \times \\
\left(\begin{array}{l}
L_{m}\left(U_{A}+U_{B}+U_{C}\right)-3 L_{m} U_{A} \\
L_{m}\left(U_{A}+U_{B}+U_{C}\right)-3 L_{m} U_{B} \\
L_{m}\left(U_{A}+U_{B}+U_{C}\right)-3 L_{m} U_{C} \\
{\left[\begin{array}{l}
\left(L_{m}\left(2 L_{R}+L_{m}-L_{S}\right)-2 L_{R} L_{S}\right) U_{A}- \\
-\left(L_{m}{ }^{2}-L_{m} L_{R}\right)\left(U_{A}+U_{B}+U_{C}\right)
\end{array}\right]} \\
L_{s}-L_{m} \\
{\left[\begin{array}{l}
\left(L_{m}\left(2 L_{R}+L_{m}-L_{S}\right)-2 L_{R} L_{S}\right) U_{B}- \\
-\left(L_{m}{ }^{2}-L_{m} L_{R}\right)\left(U_{A}+U_{B}+U_{C}\right)
\end{array}\right]} \\
L_{S}-L_{m} \\
{\left[\begin{array}{l}
\left(L_{m}\left(2 L_{R}+L_{m}-L_{S}\right)-2 L_{R} L_{S}\right) U_{C}- \\
-\left(L_{m}{ }^{2}-L_{m} L_{R}\right)\left(U_{A}+U_{B}+U_{C}\right)
\end{array}\right]}
\end{array}\right]
\end{gathered}
$$

Такая запись (13) удобна тем, что если система векторов напряжений статора симметрична, то комбинации вида $\left(U_{A}+U_{B}+U_{C}\right)$ будут равняться нулю. Здесь матрицы $\mathbf{A}_{11}, \mathbf{A}_{22}, \mathbf{A}_{12}, \mathbf{A}_{21}$ симметричные и имеют следующий вид:

$$
\begin{aligned}
& \mathbf{A}_{11}=\frac{L_{m} R_{R}\left(L_{S}-L_{m}\right)}{\left(L_{R}-L_{m}\right)\left[2 L_{R} L_{3}+\left(L_{S}+L_{R}-4 L_{m}\right) L_{m}\right]} \times \\
& \times\left(\begin{array}{ccc}
c & -1 & -1 \\
-1 & c & -1 \\
-1 & -1 & c
\end{array}\right), \\
& \mathbf{A}_{22}=\frac{L_{m} R_{S}\left(L_{R}-L_{m}\right)}{\left(L_{S}-L_{m}\right)\left[2 L_{R} L_{3}+\left(L_{S}+L_{R}-4 L_{m}\right) L_{m}\right]} \times \\
& \times\left(\begin{array}{ccc}
d & -1 & -1 \\
-1 & d & -1 \\
-1 & -1 & d
\end{array}\right),
\end{aligned}
$$

где

$$
\begin{gathered}
c=\frac{\left(2 L_{m} R_{R}-L_{R} R_{R}+L_{S} R_{R}\right) L_{m}-2 L_{R} L_{S} R_{R}}{L_{m} R_{R}\left(L_{S}-L_{m}\right)}, \\
d=\frac{\left[\left(2 L_{m}+L_{R}-L_{S}\right) L_{m}-2 L_{R} L_{S}\right] R_{S}}{L_{m} R_{S}\left(L_{R}-L_{m}\right)},
\end{gathered}
$$




$$
\begin{aligned}
& \mathbf{A}_{12}=\frac{L_{m} R_{S}}{2 L_{R} L_{S}+\left(L_{S}+L_{R}-4 L_{m}\right) L_{m}}\left(\begin{array}{ccc}
2 & -1 & -1 \\
-1 & 2 & -1 \\
-1 & -1 & 2
\end{array}\right), \\
& \mathbf{A}_{21}=\frac{L_{m} R_{R}}{2 L_{R} L_{S}-\left(L_{R}+L_{S}-4 L_{m}\right) L_{m}}\left(\begin{array}{ccc}
2 & -1 & -1 \\
-1 & 2 & -1 \\
-1 & -1 & 2
\end{array}\right) .
\end{aligned}
$$

Для вычисления корней решаем характеристическое уравнение:

$$
|\mathbf{A}-\mathbf{I} p|=0, \quad \mathbf{A}=\left(\begin{array}{ll}
\mathbf{A}_{11} & \mathbf{A}_{12} \\
\mathbf{A}_{21} & \mathbf{A}_{22}
\end{array}\right) .
$$

Здесь I - единичная матрица шестого порядка; $p$ корень характеристического уравнения.

Получаем аналитическое решение для корней характеристического уравнения:

$$
\left(\begin{array}{c}
p_{1} \\
p_{2} \\
p_{3} \\
p_{4} \\
p_{5} \\
p_{6}
\end{array}\right)=\left(\begin{array}{c}
\frac{-R_{R}}{L_{R}-L_{m}} \\
\frac{-R_{S}}{L_{S}-L_{m}} \\
-\frac{S_{1}+S_{2}+S_{3}}{S_{4}} \\
-\frac{S_{1}+S_{2}+S_{3}}{S_{4}} \\
-\frac{S_{1}+S_{2}-S_{3}}{S_{4}} \\
-\frac{S_{1}+S_{2}-S_{3}}{S_{4}}
\end{array}\right),
$$

$S_{1}=2\left(L_{R} R_{S}-L_{S} R_{R}\right) ; S_{2}=L_{m}\left(R_{S}+R_{R}\right) ;$
где $S_{3}=\sqrt{\left[\begin{array}{l}\left(L_{R} R_{S}-L_{S} R_{R}\right)^{2} 4+ \\ \left.+4 L_{m}\left(L_{S} R_{R}^{2}+L_{R} R_{S}^{2}-R_{S} R_{R}\left(L_{R}+L_{S}\right)\right)+\right] \\ +L_{m}{ }^{2}\left(R_{R}^{2}+R_{S}^{2}+34 R_{R} R_{S}\right)\end{array}\right]}$

$$
S_{4}=2\left(2 L_{R} L_{S}-L_{m}\left(L_{R}+L_{S}-4 L_{m}\right)\right) \text {. }
$$

Приведем обратную матрицу системы, построенной на основе (9) и (10):

$$
\mathbf{A}^{-1}=\left(\begin{array}{cccccc}
\frac{-L_{R}}{R_{R}} & \frac{L_{m}}{2 R_{R}} & \frac{L_{m}}{2 R_{R}} & \frac{-L_{m}}{R_{R}} & \frac{L_{m}}{2 R_{R}} & \frac{L_{m}}{2 R_{R}} \\
\frac{L_{m}}{2 R_{R}} & \frac{-L_{R}}{R_{R}} & \frac{L_{m}}{2 R_{R}} & \frac{L_{m}}{2 R_{R}} & \frac{-L_{m}}{R_{R}} & \frac{L_{m}}{2 R_{R}} \\
\frac{L_{m}}{2 R_{R}} & \frac{L_{m}}{2 R_{R}} & \frac{-L_{R}}{R_{R}} & \frac{L_{m}}{2 R_{R}} & \frac{L_{m}}{2 R_{R}} & \frac{-L_{m}}{R_{R}} \\
\frac{-L_{m}}{R_{S}} & \frac{L_{m}}{2 R_{S}} & \frac{L_{m}}{2 R_{S}} & \frac{-L_{S}}{R_{S}} & \frac{L_{m}}{2 R_{S}} & \frac{L_{m}}{2 R_{S}} \\
\frac{L_{m}}{2 R_{S}} & \frac{-L_{m}}{R_{S}} & \frac{L_{m}}{2 R_{S}} & \frac{L_{m}}{2 R_{S}} & \frac{-L_{S}}{R_{S}} & \frac{L_{m}}{2 R_{S}} \\
\frac{L_{m}}{2 R_{S}} & \frac{L_{m}}{2 R_{S}} & \frac{-L_{m}}{R_{S}} & \frac{L_{m}}{2 R_{S}} & \frac{L_{m}}{2 R_{S}} & \frac{-L_{S}}{R_{S}}
\end{array}\right)
$$

Правильность вычисления матриц А и В можно проверить, умножив обратную матрицу $\mathbf{A}^{-1}$ на вектор внешнего воздействия $\mathbf{B}$, предполагая, что внешние напряжения постоянные, например, равны единице. Легко увидеть, что при этом получаются установившиеся значения токов статора в каждой фазе как напряжения фаз, деленные на активные сопротивления статорной цепи, а токи в короткозамкнутом роторе равны нулю:

$$
-\mathbf{A}^{-1} \mathbf{B}=
$$

$$
\begin{aligned}
& =\left(\begin{array}{cccccc}
\frac{-L_{R}}{R_{R}} & \frac{L_{m}}{2 R_{R}} & \frac{L_{m}}{2 R_{R}} & \frac{-L_{m}}{R_{R}} & \frac{L_{m}}{2 R_{R}} & \frac{L_{m}}{2 R_{R}} \\
\frac{L_{m}}{2 R_{R}} & \frac{-L_{R}}{R_{R}} & \frac{L_{m}}{2 R_{R}} & \frac{L_{m}}{2 R_{R}} & \frac{-L_{m}}{R_{R}} & \frac{L_{m}}{2 R_{R}} \\
\frac{L_{m}}{2 R_{R}} & \frac{L_{m}}{2 R_{R}} & \frac{-L_{R}}{R_{R}} & \frac{L_{m}}{2 R_{R}} & \frac{L_{m}}{2 R_{R}} & \frac{-L_{m}}{R_{R}} \\
\frac{-L_{m}}{R_{S}} & \frac{L_{m}}{2 R_{S}} & \frac{L_{m}}{2 R_{S}} & \frac{-L_{S}}{R_{S}} & \frac{L_{m}}{2 R_{S}} & \frac{L_{m}}{2 R_{S}} \\
\frac{L_{m}}{2 R_{S}} & \frac{-L_{m}}{R_{S}} & \frac{L_{m}}{2 R_{S}} & \frac{L_{m}}{2 R_{S}} & \frac{-L_{S}}{R_{S}} & \frac{L_{m}}{2 R_{S}} \\
\frac{L_{m}}{2 R_{S}} & \frac{L_{m}}{2 R_{S}} & \frac{-L_{m}}{R_{S}} & \frac{L_{m}}{2 R_{S}} & \frac{L_{m}}{2 R_{S}} & \frac{-L_{S}}{R_{S}}
\end{array}\right) \times \\
& \times \frac{1}{2 L_{S} L_{R}+L_{m}\left(L_{R}+L_{S}-4 L_{m}\right)} \times \\
& \left\{\begin{array}{l}
L_{m}\left(U_{A}+U_{B}+U_{C}\right)-3 L_{m} U_{A} \\
L_{m}\left(U_{A}+U_{B}+U_{C}\right)-3 L_{m} U_{B} \\
L_{m}\left(U_{A}+U_{B}+U_{C}\right)-3 L_{C}
\end{array}\right. \\
& \frac{\left[\begin{array}{l}
\left(L_{m}\left(2 L_{R}+L_{m}-L_{S}\right)-2 L_{R} L_{S}\right) U_{A}- \\
-\left(L_{m}^{2}-L_{m} L_{R}\right)\left(U_{A}+U_{B}+U_{C}\right)
\end{array}\right]}{L_{S}-L_{m}}
\end{aligned}
$$

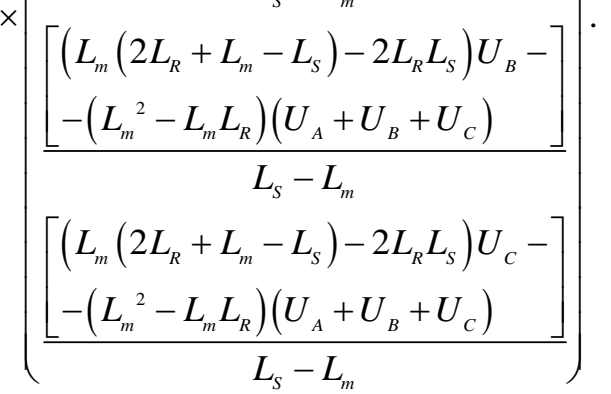

Аналитическое выражение для переменного синусоидального тока получается громоздким, поэтому приведем его в матричной форме:

$$
\left(\begin{array}{l}
i_{a \text { уст }} \\
i_{b \text { уст }} \\
i_{c \text { уст }} \\
i_{\text {A уст }} \\
i_{B \text { уст }} \\
i_{C \text { уст }}
\end{array}\right)=\operatorname{Im}\left((\mathbf{I} j \omega-\mathbf{A})^{-1} \mathbf{B} e^{j \omega t}\right)=\left(\begin{array}{c}
I_{a} \sin \left(\omega \mathrm{t}+\varphi_{a}\right) \\
I_{b} \sin \left(\omega \mathrm{t}+\varphi_{b}\right) \\
I_{c} \sin \left(\omega \mathrm{t}+\varphi_{c}\right) \\
I_{A} \sin \left(\omega \mathrm{t}+\varphi_{A}\right) \\
I_{B} \sin \left(\omega \mathrm{t}+\varphi_{B}\right) \\
I_{C} \sin \left(\omega \mathrm{t}+\varphi_{C}\right)
\end{array}\right) .
$$


Амплитуда и фаза определяются решением системы уравнений:

$$
\begin{gathered}
\left(\begin{array}{l}
I_{a} e^{j \varphi_{a}} \\
I_{b} e^{j \varphi_{b}} \\
I_{c} e^{j \varphi_{c}} \\
I_{A} e^{j \varphi_{A}} \\
I_{B} e^{j \varphi_{B}} \\
I_{C} e^{j \varphi_{C}}
\end{array}\right)=\left(\begin{array}{llllll}
d & b & b & c & b & b \\
b & d & b & b & c & b \\
b & b & d & b & b & c \\
c & b & b & d & b & b \\
b & c & b & b & d & b \\
b & b & c & b & b & d
\end{array}\right)^{-1}\left(\begin{array}{l}
0 \\
0 \\
0 \\
U_{A} \\
U_{A} a^{2} \\
U_{A} a
\end{array}\right), \\
d=R_{R}+j \omega L_{R} ; b=-j \omega \frac{L_{m}}{2} ; c=j \omega L_{m} .
\end{gathered}
$$

Здесь $a$ - оператор поворота;

$$
a=e^{j 120^{\circ}}=-1 / 2+j \sqrt{3 / 2} .
$$

$\mathrm{C}$ помощью матрицы состояния А и корней характеристического уравнения $p$ представим решение в аналитическом виде:

$$
\left(\begin{array}{c}
i_{a}(t) \\
i_{b}(t) \\
i_{c}(t) \\
i_{A}(t) \\
i_{B}(t) \\
i_{C}(t)
\end{array}\right)=\left(\begin{array}{c}
i_{a}(0) \\
i_{b}(0) \\
i_{c}(0) \\
i_{A}(0) \\
i_{B}(0) \\
i_{C}(0)
\end{array}\right) e^{\mathbf{A} t}+\int_{0}^{t} e^{\mathbf{A}(t-\tau)} \mathbf{B}(\tau) d \tau .
$$

Здесь $e^{\mathbf{A} t}-$ экспоненциальная матрица, которая представляется в виде

$$
\begin{gathered}
e^{\mathbf{A} t}=\mathbf{I} \alpha_{0}(t)+\mathbf{A} \alpha_{1}(t)+\mathbf{A}^{2} \alpha_{2}(t)+ \\
+\mathbf{A}^{3} \alpha_{3}(t)+\mathbf{A}^{4} \alpha_{4}(t)+\mathbf{A}^{5} \alpha_{5}(t) .
\end{gathered}
$$

Коэффициенты разложения $\alpha_{0}(t), \alpha_{1}(t), \ldots \alpha_{6}(t)$ определяются корнями характеристического уравнения:

$$
\begin{gathered}
\alpha(t)=\mathbf{P}^{-1} \cdot \exp (p t) \Rightarrow\left(\begin{array}{l}
\alpha_{0}(t) \\
\alpha_{1}(t) \\
\alpha_{2}(t) \\
\alpha_{3}(t) \\
\alpha_{4}(t) \\
\alpha_{5}(t)
\end{array}\right)= \\
\left.=\left(\begin{array}{lllllll}
1 & p_{1} & p_{1}^{2} & p_{1}^{3} & p_{1}^{4} & p_{1}^{5} \\
1 & p_{2} & p_{2}^{2} & p_{2}^{3} & p_{2}^{4} & p_{2}^{5} \\
1 & p_{3} & p_{3}^{2} & p_{3}^{3} & p_{3}^{4} & p_{3}^{5} \\
1 & p_{4} & p_{4}^{2} & p_{4}^{3} & p_{4}^{4} & p_{4}^{5} \\
1 & p_{5} & p_{5}^{2} & p_{5}^{3} & p_{5}^{4} & p_{5}^{5} \\
1 & p_{6} & p_{6}^{2} & p_{6}^{3} & p_{6}^{4} & p_{6}^{5}
\end{array}\right)^{j p_{1} t}\right)\left(\begin{array}{l}
e^{j p_{2} t} \\
e^{j p_{3} t} \\
e^{j p_{4} t} \\
e^{j p_{5} t} \\
e^{j p_{6} t}
\end{array}\right) .
\end{gathered}
$$

Следует учесть, что, если размерность обратной матрицы выше трех, при наличии кратных корней, то она может быть сингулярной. В этом случае следует сдвинуть ее спектр на малую величину, например $\lambda^{-10}$ :

$$
\alpha(t)=(\mathbf{P}-\mathbf{I} \lambda)^{-1} \cdot \exp (p t)
$$

\section{Имитационное моделирование асинхронной} $n$-фазной машины

Моделирование $n$-фазной асинхронной машины производилось в программной среде Matlab Simulink2021a при использовании стандартных библиотек. Для исследования был выбран асинхронный электродвигатель типа АДМ100S4У3 с параметрами, показанными в табл. 1.

Таблица 1. Заводские параметры асинхронного двигателя типа АДМ100S4У3

Table 1. Factory parameters of ADM100S4U3 type induction motor

\begin{tabular}{|c|c|c|c|c|}
\hline$R_{1}$ & $R_{2}$ & $L_{1}$ & $L_{2}^{\prime}$ & $L_{\mathrm{m}}$ \\
\hline \multicolumn{2}{|c|}{$\mathrm{OM} / \mathrm{Ohm}$} & \multicolumn{3}{|c|}{$\Gamma_{\mathrm{H}} / \mathrm{H}$} \\
\hline 1,851 & 1,118 & 0,011 & 0,014 & 0,2138 \\
\hline
\end{tabular}

\begin{tabular}{|c|c|c|c|c|c|c|}
\hline $\begin{array}{c}P_{\mathrm{H}}, \mathrm{\kappa BT} \\
P_{\text {nom }}, \\
\mathrm{kW}\end{array}$ & $\begin{array}{c}I_{\mathrm{H}}, \mathrm{A} \\
I_{\text {nom }}, \\
\mathrm{A}\end{array}$ & $\begin{array}{c}n_{\mathrm{H}}, \\
\text { об/мин } \\
n_{\text {nom }}, \mathrm{rpm}\end{array}$ & $\begin{array}{c}\eta, \\
\%\end{array}$ & $\begin{array}{c}\cos \\
\varphi\end{array}$ & $\begin{array}{c}U_{\phi}, \mathrm{B} \\
U_{L \text { to } G}, \\
\mathrm{~V}\end{array}$ & $\begin{array}{c}J, \\
\mathrm{\kappa} \cdot \mathrm{M}^{2} \\
\mathrm{~kg} \cdot \mathrm{m}^{2}\end{array}$ \\
\hline 3 & 7,17 & 1410 & 82 & 0,82 & 220 & 0,0100 \\
\hline
\end{tabular}

В табл. 2 представлены параметры схемы замещения АДM100S4У3, которые были определены косвенным методом.

Таблица 2. Параметры схемы замещения асинхронного двигателя типа АДМ100S4У3

Table 2. Simulation parameters of ADM100S4U3 type induction motor

При изменении числа фаз необходимо учитывать, что меняется максимальная приведенная взаимная индуктивность между фазами статора и ротора:

$$
L_{m 1}=L_{m} \cdot \frac{2}{n},
$$

где $n$ - число фаз статора и ротора.

Число стержней для исследуемого двигателя составляет $n=28$.

Схема имитационной модели в Matlab Simulink c основными элементами показана на рис. 5. Для обеспечения оптимальных расчетных показателей был выбран метод Рунге-Кутты 4-го порядка с фиксированными шагом расчета 0,00002 c.

В блок 1 подаются трехфазные синусоидальные напряжения (4)-(6), которые сдвинуты на 120 градусов. Затем с помощью блока - 2 преобразуются мгновенные напряжения из трехфазной системы координат в многофазную по выражению (3) и формируется вектор входных воздействий (2). В блоке -4 осуществляется расчет токов статора и ротора на основе системы дифференциальных уравнений (7), а в блоке - 5 момента двигателя с помощью соотношения (8). Блоки - 2, 4 и 5 построены на основе программных функций.

Далее на основе полученного момента двигателя определяется угловая скорость вала ротора (блок 6) по дифференциальному уравнению (9). Данные о скорости используются для определения угла, который необходим для вычисления взаимных индуктивностей в блоке -4 .

Задание повреждений в стержнях ротора реализуется путем увеличения сопротивления в блоке -3 . 


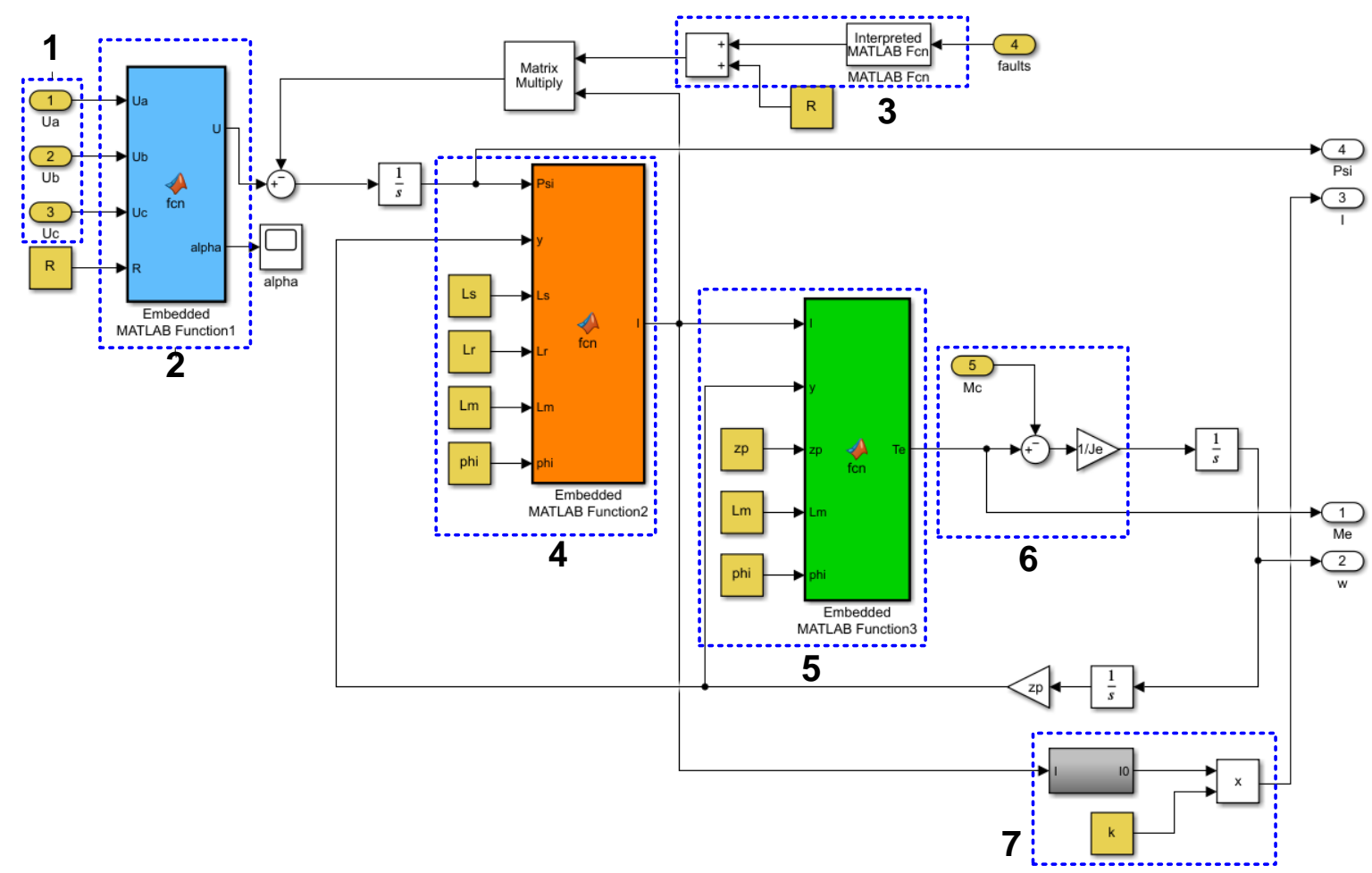

Pис. 5. Имитачионная модель асинхронного двигателя в Matlab Simulink

Fig. 5. Simulation model of an induction motor in Matlab Simulink

Для верификации имитационной модели и математического описания $n$-фазной машины использовались аналитические выражения при неподвижном роторе. Такой подход исключает из решений расчётную погрешность при использовании численных методов и дает возможность оценить адекватность результатов. Ввиду определенных сложностей, связанных с формированием аналитических выражений для $n$ фазной машины при неподвижном роторе, взят частный случай при $n=3$.

В качестве критерия для оценки работоспособности предложенной имитационной модели $n$-фазной машины выбрана огибающая токов статора:

$$
I_{0}(t)=\sqrt{I_{A}^{2}(t)+I_{B}^{2}(t)+I_{C}^{2}(t)} .
$$

На основе фазных статорных токов проведены вычисления огибающих по аналитическим уравнениям, затем получены данные с помощью имитационной модели (рис. 5) при $n=3$, что соответствует описанию асинхронной машины в естественной системе координат и при $n=28$, а также для асинхронной машины в неподвижной $\alpha, \beta$ системе координат. Имитация неподвижного ротора для многофазной машины при $n=3, n=28$ и для двухфазной модели осуществлялась путем задания угловой скорости ротора равной нулю $\omega=0$.

Для корректного сравнения огибающих токов статора все они были приведены к трехфазной системе координат с применением масштабирующего коэффициента $k$ в блоке -7 , который определялся по выражению (8).

Приведем пример аналитического определения токов статора и ротора для асинхронного двигателя при неподвижном роторе с параметрами двигателя АДM100S4У3 (табл. 2). Внешнее воздействие в статорной цепи будем считать симметричным, начальные условия задачи примем нулевыми

$$
i_{a}(0)=i_{b}(0)=i_{c}(0)=i_{A}(0)=i_{B}(0)=i_{C}(0)=0 .
$$

С учетом условия задачи $U_{A}(t)+U_{B}(t)+U_{C}(t)=0$ получаем на основе (13):

$$
\begin{aligned}
& \mathbf{B}(t)=\frac{1}{2 L_{S} L_{R}+L_{m}\left(L_{R}+L_{S}-4 L_{m}\right)} \times \\
& \times\left(\begin{array}{l}
-3 L_{m} \cdot U_{A} \\
-3 L_{m} \cdot U_{B} \\
-3 L_{m} \cdot U_{C} \\
\frac{\left(L_{m}\left(2 L_{R}+L_{m}-L_{S}\right)-2 L_{R} L_{S}\right) U_{A}}{L_{S}-L_{m}} \\
\frac{\left(L_{m}\left(2 L_{R}+L_{m}-L_{S}\right)-2 L_{R} L_{S}\right) U_{B}}{L_{S}-L_{m}} \\
\frac{\left(L_{m}\left(2 L_{R}+M-L_{S}\right)-2 L_{R} L_{S}\right) U_{C}}{L_{S}-L_{m}}
\end{array}\right)= \\
& =\left(\begin{array}{c}
-39,246 U_{A}(t) \\
-39,246 U_{B}(t) \\
-39,246 U_{C}(t) \\
40,959 U_{A}(t) \\
40,959 U_{B}(t) \\
40,959 U_{C}(t)
\end{array}\right) .
\end{aligned}
$$

Корни характеристического уравнения будут: 


$$
\begin{gathered}
p_{1}=-159,714 \frac{1}{c}, \quad p_{2}=-168,273 \frac{1}{c}, \\
p_{3}=p_{4}=-163,482 \frac{1}{c}, \quad p_{5}=p_{6}=-3,098 \frac{1}{c} .
\end{gathered}
$$

Коэффициенты разложения экспоненциальной матрицы определяются системой:

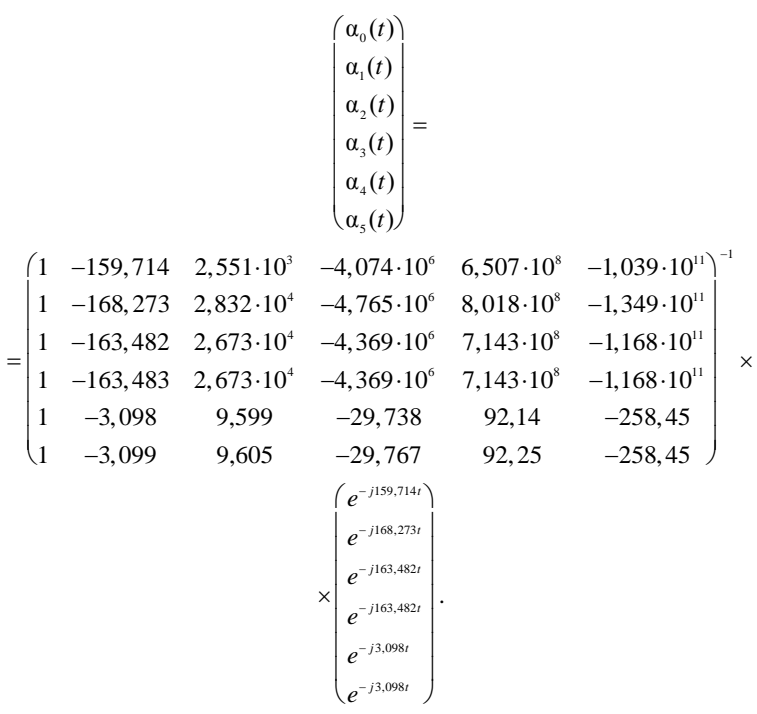

После вычисления интеграла (14) с нулевыми начальными условиями получаем графические зави- симости, представленные на интервале длительностью в три периода для токов ротора (рис. 6) и статора (рис. 7), где пунктирными линиями показаны значения для статического режима.

На рис. 8 показаны огибающие токов статора при неподвижном роторе, полученные аналитическим и численным способом. Из данных кривых видно, что огибающие токов 2, 3, 4, полученные численным методом, имеют схожий вид. Наибольшее расхождение наблюдается с кривой тока 1 , которая получена аналитическим способом. На основе результатов рис. 8 можно сделать вывод, что погрешность для $n$-фазной модели находится на приемлемом уровне и эту модель можно использовать для дальнейших исследований.

Исследование обрыва стержней в роторе для $n$ фазной асинхронной машины осуществлялось путем введения добавочного сопротивления в уравнения для соответствующей фазы. Данная модель позволяет задавать количество обрывов и место их расположения.

При появлении аварийных стержней в огибающей токов статора наблюдаются гармонические колебания (рис.9), которые зависят от текущей нагрузки и от процентного соотношения дефектных стержней и нормальных. На рис. 9 показаны кривые токов статора в различных режимах работы (пуск, холостой ход, нагрузка) при разных состояниях роторной цепи $(1,2$, 3, 4 оборванных стержня).

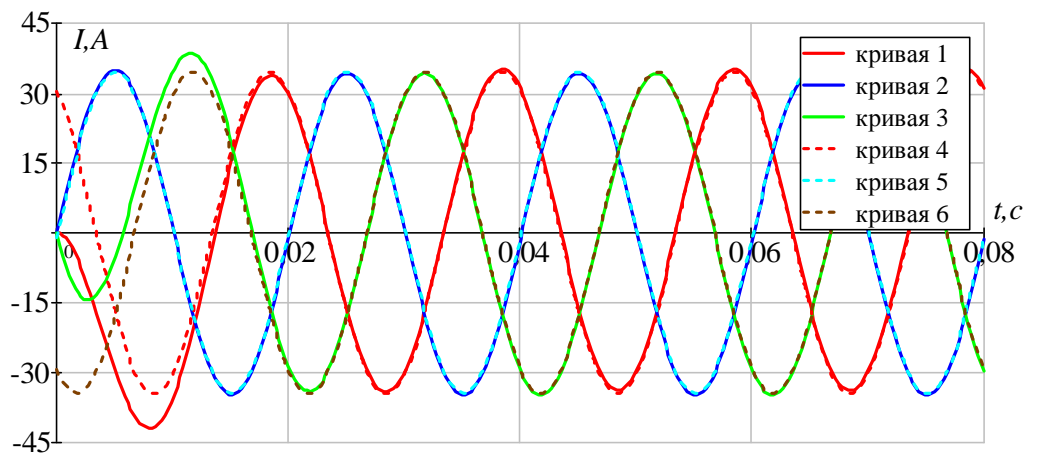

Pис. 6. Кривые 1-3 - токи ротора фазы а, b, с в переходном проиессе соответственно. Кривые 4-6 - токи ротора фазы $a, b$, с в установившемся проиессе соответственно

Fig. 6. Curves 1-3 are rotor currents of the phase $a, b$, $c$ in transient process, respectively. Curves 4-6 are rotor currents of phases $a, b, c$ in a steady process, respectively

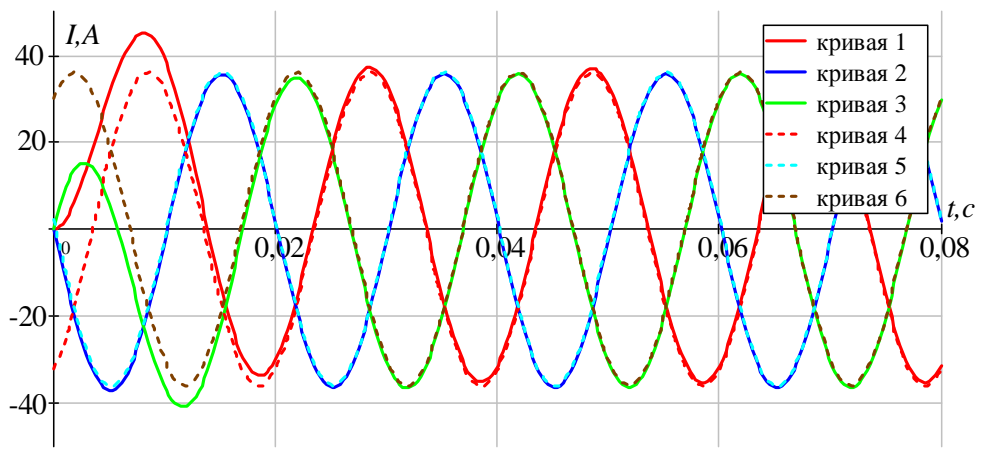

Pис. 7. Кривые 1-3-токи статора фазы A, B, С в переходном проиессе соответственно. Кривые 4-6 - токи ротора фазы $A, B, C$ в установившемся прочессе соответственно

Fig. 7. Curves 1-3 are stator currents of phases $A, B, C$ in the transient process, respectively. Curves 4-6 are rotor currents of phases $A, B, C$ in a steady process, respectively 


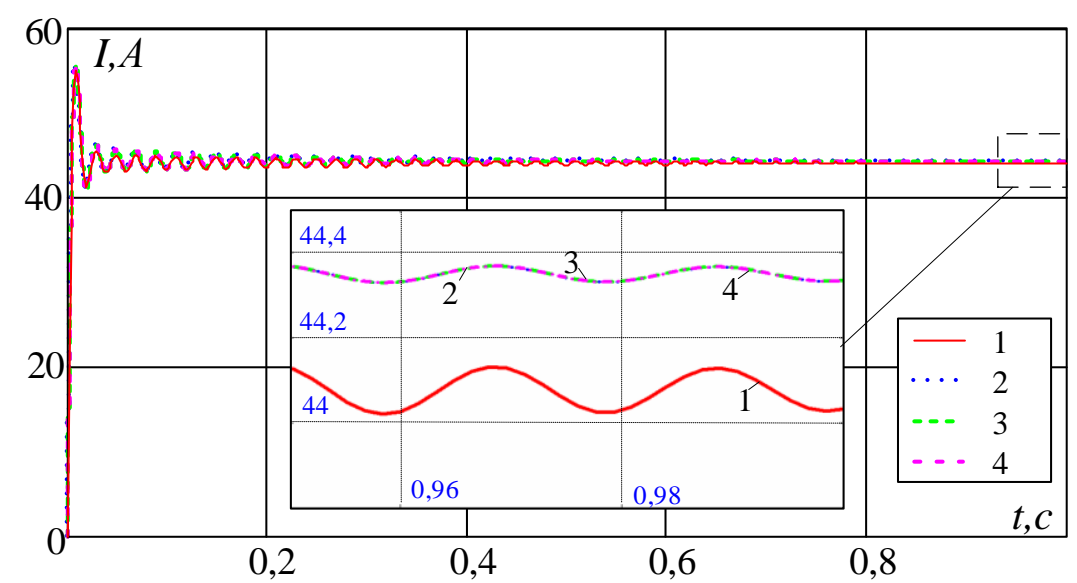

Рис. 8. Огибающие токов статора при неподвижном роторе: 1 - эталонные значения, полученные аналитическим способом; 2 - кривая, полученная численным способом для многофазной машины при n=3; 3 - Кривая, вычисленная численным способом для двухфазной машины в неподвижной $\alpha$, $\beta$ системе координат; 4 - кривая, полученная численным способом для многофазной машины при $n=28$

Fig. 8. Stator current envelopes: 1 - reference values obtained analytically; 2 - curve calculated numerically for a multiphase machine with $n=3 ; 3$ - curve calculated numerically for a two-phase machine in a fixed $\alpha$, $\beta$ coordinate system; 4 - curve calculated numerically for a multiphase machine with $n=28$

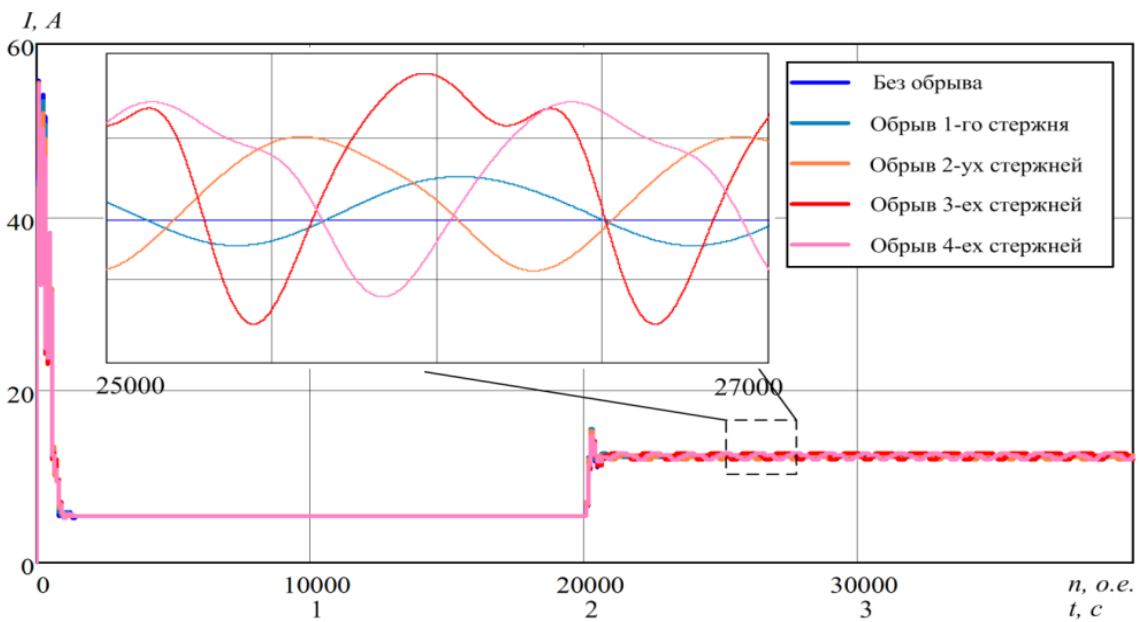

Pис. 9. Огибающие токов статора в различных режимах

Fig. 9. Stator current envelope in different modes

Из рис. 9 видно, что гармонические колебания при обрыве стержней имеют определённую частоту и амплитуду. Анализируя эти составляющие, а также их форму, можно сделать выводы о текущем состоянии двигателя. Огибающая токов статора содержит диагностический признак, позволяющий с определенной точностью определить обрыв стержня в роторе.

На данный момент существуют различные подходы и методы, которые позволяют выявлять из токов, напряжений и других информационных сигналов нужный диагностический признак, а также получать оценку текущего состояния электрической машины [16-21]. Данные диагностические системы строятся на основе использования определенного математического аппарата, который дает возможность сформировать численную оценку. Предложенная $n$-фазная модель позволяет на основе результатов моделирования выявить работоспособный подход для диагностики обрыва стержней в роторной цепи.

\section{Заключение}

Разработана $n$-фазная математическая модель асинхронной машины, которая позволяет с приемлемой точностью исследовать работу двигателя как в нормальном состоянии, так и при обрыве стержней. Описание процессов в статоре и роторе с одной и той же размерностью системы координат приводит к значительному увеличению времени моделирования при большом количестве стержней.

Для исследования режимов работы асинхронной машины при неподвижном роторе предложено решение, полученное аналитическим способом. Данный подход существенно снижает требованиям к вычислительным мощностям.

Адекватность математического описания $n$-фазной машины подтверждено с помощью огибающих токов статора, которые были получены при помощи аналитических выражений и $n$-фазной модели. 
Показано, что кривая огибающей токов статора изменяет свою форму в зависимости от количества исключенных из расчетов стержней ротора математи-

\section{СПИСОК ЛИТЕРАТУРЫ}

1. Сафин Н.Р. Совершенствование методики токовой диагностики асинхронных двигателей с короткозамкнутым ротором: дис. ... канд. техн. наук. - Екатеринбург, 2017. - 152 с.

2. Зюзев А.М., Метельков В.П. О проблеме перегрева обмотки ротора асинхронных двигателей в пусковых режимах высокоинерционных электроприводов нефтегазовой и горной промышленности // Известия Томского политехнического университета. Инжиниринг георесурсов. - 2018. - Т. 329. - № 7. C. $96-103$.

3. Ouadah M., Touhami O., Ibtiouen R. Improvement of the detection of the defect squirrel cage rotor by the study of additional components of the space harmonics // Electrical Engineering. - 2018. - V. 100. - P. 2485-2497.

4. Kumar P., Hati A.S. Deep convolutional neural network based on adaptive gradient optimizer for fault detection in SCIM // ISA Transactions. - 2021. - V. 111. - P. 350-359.

5. Abdi Monfared O., Doroudi A., Darvishi A. Diagnosis of rotor broken bars faults in squirrel cage induction motor using continuous wavelet transform // COMPEL - The International Journal for Computation and Mathematics in Electrical and Electronic Engineering. - 2019. - V. 38. - № 1. - P. 167-182.

6. De La Barrera P.M., Otero M., Schallschmidt T. Active broken rotor bar diagnosis in induction motor drives // IEEE Transactions on Industrial Electronics. - 2021. - V. 68. - № 8 - P. 7556-7566.

7. Fault diagnosis of mine asynchronous motor based on MEEMD energy entropy and ANN / Z. Yang, C. Kong, Y. Wang, X. Rong, L. Wei // Computers and Electrical Engineering. - 2021. - V. 92. P. 568-575.

8. An analytical model for squirrel cage induction machine with broken rotor bars derived based on the multiple coupled circuit theory and the winding function approach / Q. Fu, Q. Guo, W. Hang, W. Cui, L. Zhao // International Journal of Circuit Theory and Applications. - 2021. - V. 49. - № 6. - P. 1633-1658.

9. Лавренов Е.О. Методы компенсации влияния электрической несимметрии на механический момент индукционного двигателя // Известия Томского политехнического университета. Инжиниринг георесурсов. - 2016. - Т. 327. - № 1. - С. 72-78.

10. Зюзев А.М., Метельков В.П. Аналитический метод оценки нагрева обмотки ротора высоковольтных асинхронных двигателей в пусковых режимах // Электротехнические системы и комплексы. - 2017. - Т. 34 - № 1. - С. 60-67.

11. Tarek A., Yassine K., Ahmed T. Modeling and diagnostics of inductions machines under rotor failure // Automatic Control and System Engineering journal. - 2007. - V. 7. - № 2. - P. 9-18. ческой модели и позволяет выявить диагностический признак, который характеризует состояние электродвигателя.

12. Математическое моделирование асинхронных двигателей при повреждениях стержней короткозамкнутого ротора / В.Ф. Сивокобыленко, В.А. Павлюков, В.П. Сердюков, В.И. Бондаренко, С.П. Яременко // ДонНТУ. - 2009. - Т. 158. - № 9. С. $222-226$.

13. Виноградов А.Б. Векторное управление электроприводами переменного тока. - Иваново: ГОУВПО «Ивановский государственный энергетический университет имени В.И. Ленина», 2008. - 298 c.

14. Новожилов А.Н., Потапенко А.О., Новожилов Т.А. Моделирование процессов в асинхронном двигателе с поврежденным стержнем короткозамкнутого ротора в режиме выбега // Электротехника. - 2017. - № 1. - С. 2-6.

15. Mathematical model of induction motor with series-connected stator and rotor windings /

16. Funk T.A., Usynin Yu.S., Grebnev A.I., Ponosov D.A. // Bulletin of South Ural State University. Series: Power Engineering. 2017. - V. 17. - № 1. - P. 77-87.

17. Choudhary A., Goyal D., Shimi S.L. Condition monitoring and fault diagnosis of induction motors: a review // Archives of Computational Methods in Engineering. - 2019. - V. - 26. P. 1221-1238

18. Induction machine model with space harmonics for fault diagnosis based on the convolution theorem / J. Martinez-Roman, R. PuchePanadero, M. Pineda-Sanchez, J. Perez-Cruz, M. Riera-Guasp // International Journal of Electrical Power and Energy Systems. 2018. - V. 100. - P. 463-481.

19. The Matlab Simulink model of transformer winding when its diagnisis by short duration puls takes place / V.A. Kolchanova, Y.N. Isaev, S.S. Tarasenko, A.I. Filkov // Proceedings of 2014 International Conference on Mechanical Engineering, Automation and Control Systems. - Tomsk: Institute of Electrical and Electronics Engineers Inc., 2014. - P. 1-4.

20. Duda A., Sulowicz M. A new effective method of induction machine condition assessment based on zero-sequence voltage (ZSV) symptoms // Energies. - 2020. - V. 13. - P. 1-25.

21. Функциональная вейвлет-диагностика состояния обмоток роторов трехфазных электрических машин по статорным токам / В.В. Тимошкин, Т.А. Глазырина, В.И. Полищук, А.С. Глазырин // Электричество. - 2012. - № 6. - С. 42-46.

Поступила 13.10.2021 2.

\section{Информация об авторах}

Глазырин А.C., доктор технических наук, профессор отделения электроэнергетики и электротехники Инженерной школы энергетики Национального исследовательского Томского политехнического университета; профессор Института нефти и газа Югорского государственного университета.

Полищук В.И., доктор технических наук, профессор кафедры электроснабжения промышленных предприятий, Алтайский государственный технический университет им. И.И. Ползунова.

Tuмошкин B.B., кандидат технических наук, доцент отделения электроэнергетики и электротехники Инженерной школы энергетики Национального исследовательского Томского политехнического университета.

Баннов Д.М., инженер кафедры электрических станций Электротехнического факультета, Самарский государственный технический университет.

Исаев Ю.Н., доктор физико-математических наук, профессор отделения электроэнергетики и электротехники Инженерной школы энергетики, профессор отделения автоматизации и робототехники Инженерной школы информационных технологий и робототехники Национального исследовательского Томского политехнического университета.

Антяскин Д.И., инженер-электроник, ООО «Комплексные автоматизированные системы».

Кладиев С.H., кандидат технических наук, доцент отделения электроэнергетики и электротехники Инженерной школы энергетики Национального исследовательского Томского политехнического университета. 
Фuлипас A.A., кандидат технических наук, доцент отделения автоматизации и робототехники Инженерной школы информационных технологий и робототехники Национального исследовательского Томского политехнического университета.

Ланграф С.В., кандидат технических наук, руководитель сектора разработки встроенного ПО, ООО «НПФ Мехатроника-Про».

Котин Д.А., кандидат технических наук, заведующий кафедрой электропривода и автоматизации промышленных установок Новосибирского государственного технического университета.

Ковалев В.3., доктор технических наук, профессор Института нефти и газа Югорского государственного университета. 
UDC 621.313 .33

\section{MODELLING OF INDUCTION MOTOR IN MULTIPHASE COORDINATE SYSTEM FOR ASYMMETRY ROTOR CAGE}

\author{
Alexander S. Glazyrin 1,2 , \\ asglazyrin@tpu.ru
}

Vladimir I. Polishchuk ${ }^{3}$, polischuk_vi@mail.ru

Vadim V. Timoshkin ${ }^{1}$, tim_vv@mail.ru

Dmitry M. Bannov4, bannov.dm@samgtu.ru

Yusup N. Isaev'1, isaev_yusup@mail.ru

Dmitriy I. Antyaskin ${ }^{5}$, antyaskin.dmitriy@gmail.com

1 National Research Tomsk Polytechnic University, 30, Lenin avenue, Tomsk, 634050, Russia.

2 Yugra State University, 16, Chekhov street, Khanty-Mansiysk, 628012, Russia.

3 Polzunov Altai State Technical University, Lenin avenue, 46, Lenin avenue, Barnaul, 656038, Russia.

${ }^{4}$ Samara State Technical University, 224, Molodogvardeyskaya street, Samara, 443100, Russia.

5 Integrated automated systems, 240a, Frunze avenue, Tomsk, 634021, Russia.

${ }^{6}$ NPF Mekhatronica-Pro LTD, 119E, Frunze avenue, Tomsk, 634021, Russia.

7 Novosibirsk State Technical University, 20, K. Marx avenue, Novosibirsk, 630073, Russia.

\author{
Sergey N. Kladiev ${ }^{1}$, \\ kladiev@tpu.ru
}

\author{
Alexander A. Filipas', \\ filipas@tpu.ru
}

Sergey V. Langraf6,
sergeylgrf@gmail.com

Denis A. Kotin? d.kotin@corp.nstu.ru

Vladimir Z. Kovalev², vz_kovalev@mail.ru

Relevance of the study is caused by an urgent need in theoretical justification and practical development of selective methods of diagnosing complex internal damages of powerful high-voltage squirrel-cage induction motors, which are one of the main elements of responsible mechanisms of all technological processes in fuel and energy complex. As a rule, specificity of work of high-voltage squirrel-cage motors in fuel and energy complex, both at a stage of extraction and transportation of georesources, and at a stage of their processing, are heavy conditions of start-up, especially it is expressed for critical mechanisms of thermal power plants (feed pumps, mills, crushers, smoke exhausters, blowers, etc.). Failures of squirrel-cage induction motors of critical mechanisms at thermal power plants lead to either shutdown of a power unit or at least to reduction of a level of electric power generation. At the same time, despite the gravest and, as a rule, irreversible consequences, there are no protections against such damage as defects in short-circuited winding of squirrel-cage induction motor rotor and such damage is detected only during overhaul. Broken-rotor-bar in the initial stage is latent and is a trigger for the development of more dangerous emergency modes. Lack of tested technical means for diagnostics of this type of damage is first of all related to insufficient formalization of mathematical description of processes in squirrel-cage induction motor when defects in rotor winding occur. Object: high voltage squirrel-cage induction AC motors.

The aim of the research is to perform modeling of induction machine with electrical asymmetry rotor, adequately reflecting physical processes in the machine when damage occurs in the rotor cage.

Methods. Theoretical research methods were used to solve the problem. They include: the theory of electrical machines, numerical methods. Simulation modeling was performed in MatLab environment, and mathematical data processing in MathCad package.

Results. An n-phase simulation model of an induction motor has been developed, which allows us to investigate the broken rotor bar in a squirrel-cage. Proposed analytical expressions, which describe the motor operation when the rotor is stationary.

Key words:

Induction motor, mathematical model, squirrel-cage, broken rotor bar, technical condition. 


\section{REFERENCES}

1. Safin N.R. Sovershenstvovanie metodiki tokovoy diagnostiki asinkhronnykh dvigateley s korotkozamknutym rotorom. Dis. Kand. nauk [Improvement of the method of current diagnostics of inductions motors with squirrel-cage rotor. Cand. Diss.]. Ekaterinburg, 2017. 152 p.

2. Zyuzev V.P., Metelkov Problem of induction motors rotor winding overheating in starting modes of high-inertia electric drives of oil and mining industry. Bulletin of the Tomsk Polytechnic University. Geo Assets Engineering, 2018, vol. 329, no. 7, pp. 96-103. In Rus.

3. Ouadah M., Touhami O., Ibtiouen R. Improvement of the detection of the defect squirrel cage rotor by the study of additional components of the space harmonics. Electrical Engineering, 2018, vol. 100, pp. 2485-2497.

4. Kumar P., Hati A.S. Deep convolutional neural network based on adaptive gradient optimizer for fault detection in SCIM. ISA Transactions, 2021, vol. 111, pp. 350-359.

5. Abdi Monfared O., Doroudi A., Darvishi A. Diagnosis of rotor broken bars faults in squirrel cage induction motor using continuous wavelet transform. COMPEL - The International Journal for Computation and Mathematics in Electrical and Electronic Engineering, 2019, vol. 38, no. 1, pp. 167-182.

6. De La Barrera P.M., Otero M., Schallschmidt T. Active broken rotor bar diagnosis in induction motor drives. IEEE Transactions on Industrial Electronics, 2021, vol. 68, no. 8, pp. 7556-7566.

7. Yang Z., Kong C., Wang Y., Rong X., Wei L. Fault diagnosis of mine asynchronous motor based on MEEMD energy entropy and ANN. Computers and Electrical Engineering, 2021, vol. 92, pp. 568-575.

8. Fu Q., Guo Q., Hang W., Cui W., Zhao L. An analytical model for squirrel cage induction machine with broken rotor bars derived based on the multiple coupled circuit theory and the winding function approach. International Journal of Circuit Theory and Applications, 2021, vol. 49, no. 6, pp. 1633-1658.

9. Lavrenov E.O. Compensation methods of electrical asymmetry effect on induction motor moment. Bulletin of the Tomsk Polytechnic University. Geo Assets Engineering, 2016, vol. 327, no. 1, pp. 72-78. In Rus.

10. Zyuzev A.M., Metelkov V.P Analytical method for evaluation of high-voltage induction motor rotor winding heating at start-up mode. Elektrotekhnicheskie sistemy i kompleksy, 2016, vol. 34, no. 1, pp. 60-67. In Rus.

11. Tarek A., Yassine K., Ahmed T. Modeling and diagnostics of inductions machines under rotor failure. Automatic Control and System Engineering journal, 2007, vol. 7, no. 2, pp 9-18.
12. Sivokobylenko V.F., Pavlyukov V.A., Serdyukov V.P., Bondarenko V.I., Yaremenko S.P. Matematicheskoe modelirovanie asinkhronnykh dvigateley pri povrezhdeniyakh sterzhney korotkozamknutogo rotora [Mathematical modeling of inductions motors in case of broken rotor bar]. DonNTU, 2009, vol. 158, no. 9, pp. 222-226.

13. Vinogradov A.B. Vektornoe upravlenie elektroprivodami peremennogo toka [Vector control of AC drives]. Ivanovo, Ivanovo State Power Engineering University named after V.I. Lenin Publ., 2008. 298 p.

14. Novozhilov A.N., Potapenko A.O., Novozhilov T.A. Modeling of processes in an asynchronous motor with a damaged squirrel cage rotor bar in the coasting mode. Electrical engineering, 2017, no. 1, pp. 2-6. In Rus.

15. Funk T.A., Usynin Yu.S., Grebnev A.I., Ponosov D.A. Mathematical model of induction motor with series-connected stator and rotor windings. Bulletin of South Ural State University. Series: Power Engineering, 2017, vol. 17, no 1, pp. 77-87.

16. Choudhary A., Goyal D., Shimi S.L. Condition monitoring and fault diagnosis of induction motors: a review. Archives of Computational Methods in Engineering, 2019, vol. 26, pp. 1221-1238.

17. Martinez-Roman J., Puche-Panadero R., Pineda-Sanchez M., Perez-Cruz J., Riera-Guasp M. Induction machine model with space harmonics for fault diagnosis based on the convolution theorem. International Journal of Electrical Power and Energy Systems, 2018, vol. 100, pp. 463-481.

18. Asad B., Vaimann T., Belahcen A., Kallaste A., Rassõlkin A., Iqbal M.N. Modified winding function-based model of squirrel cage induction motor for fault diagnostics. IET Electric Power Applications, 2020, vol. 14, no. 9, pp. 1722-1734.

19. Duda A., Sulowicz M. A new effective method of induction machine condition assessment based on zero-sequence voltage (ZSV) symptoms. Energies, 2020, vol. 13, pp. 1-20.

20. Kolchanova V.A., Isaev Y.N., Tarasenko S.S., Filkov A.I. The Matlab Simulink model of transformer winding when its diagnisis by short duration puls takes place. Proceedings of 2014 International Conference on Mechanical Engineering, Automation and Control Systems. Tomsk, Institute of Electrical and Electronics Engineers Inc., 2014. pp. 1-4.

21. Timoshkin V.V., Glazyrina T.A., Polishchuk V.I., Glazyrin A.S Functional Wavelet diagnostics of three-phase electric machine rotor windings condition by stator currents. Elektrichestvo, 2012, no. 6, pp. 42-46. In Rus.

Received: 13 October 2021.

Information about the authors

Alexander S. Glazyrin, Dr. Sc., professor, National Research Tomsk Polytechnic University; professor, Yugra State University.

Vladimir I. Polishchuk, Dr. Sc., professor, Polzunov Altai State Technical University.

Vadim V. Timoshkin, Cand. Sc., associate professor, National Research Tomsk Polytechnic University.

Dmitry M. Bannov, engineer, Samara State Technical University.

Yusup N. Isaev, Dr. Sc., professor, National Research Tomsk Polytechnic University.

Sergey N. Kladiev, Cand. Sc., associate professor, National Research Tomsk Polytechnic University.

Dmitriy I. Antyaskin, electronic engineer, Integrated automated systems.

Alexander A. Filipas, Cand. Sc., associate professor, National Research Tomsk Polytechnic University.

Sergey $V$. Langraf, head of the embedded software development sector, NPF Mekhatronica-Pro Ltd.

Denis A. Kotin, Cand. Sc., head of the department, Novosibirsk State Technical University.

Vladimir Z. Kovalev, Dr. Sc., professor, Yugra State University. 